\title{
Gene by Sex Interaction for Measures of Obesity in the Framingham Heart Study
}

\author{
Ashlee M. Benjamin, ${ }^{1}$ Sunil Suchindran, ${ }^{1}$ Kaela Pearce, ${ }^{1}$ Jennifer Rowell, ${ }^{2}$ Lillian F. Lien, ${ }^{2}$ \\ John R. Guyton, ${ }^{2}$ and Jeanette J. McCarthy ${ }^{1,3}$ \\ ${ }^{1}$ Duke Institute for Genome Sciences and Policy, Duke University, Durham, NC 27708, USA \\ ${ }^{2}$ Division of Endocrinology, Metabolism and Nutrition, Department of Medicine, Duke University Medical Center, \\ Durham, NC 27710, USA \\ ${ }^{3}$ Department of Community and Family Medicine, Duke University Medical Center, Durham, NC 27710, USA
}

Correspondence should be addressed to Jeanette J. McCarthy, jeanette.mccarthy@duke.edu

Received 14 September 2010; Revised 17 November 2010; Accepted 22 November 2010

Academic Editor: Yvon Chagnon

Copyright $(\odot 2011$ Ashlee M. Benjamin et al. This is an open access article distributed under the Creative Commons Attribution License, which permits unrestricted use, distribution, and reproduction in any medium, provided the original work is properly cited.

Obesity is an increasingly prevalent and severe health concern with a substantial heritable component and marked sex differences. We sought to determine if the effect of genetic variants also differed by sex by performing a genome-wide association study modeling the effect of genotype-by-sex interaction on obesity phenotypes. Genotype data from individuals in the Framingham Heart Study Offspring cohort were analyzed across five exams. Although no variants showed genome-wide significant gene-by-sex interaction in any individual exam, four polymorphisms displayed a consistent BMI association ( $P$-values .00186 to .00010) across all five exams. These variants were clustered downstream of LYPLAL1, which encodes a lipase/esterase expressed in adipose tissue, a locus previously identified as having sex-specific effects on central obesity. Primary effects in males were in the opposite direction from females and were replicated in Framingham Generation 3. Our data support a sex-influenced association between genetic variation at the LYPLAL1 locus and obesity-related traits.

\section{Introduction}

Overweight and obesity present a major public health challenge in the developed world and are a primary focus of preventive healthcare. Rates of both overall adiposity, measured by body mass index (BMI), as well as central (intraabdominal) adiposity, measured by waist circumference (WC) or waist to hip ratio (WHR) have been steadily rising during the past several decades, accompanied by increased rates of diabetes mellitus, cardiovascular disease, and other morbidities [1]. In the United States, regional, racial, and sex differences in adiposity have been noted, but the patterns are complex and changing over time [1]. According to U.S. national health survey data, men on average have had a higher BMI than women, but since the mid 1990s the average BMI in women has been higher than men [2]. Men also tend to have larger abdominal girth than women, and this disparity has persisted over time $[3,4]$.
Obesity is a heritable trait and recent genome-wide association studies have identified dozens of loci influencing measures of adiposity [5-8]. Sex differences in the heritability of obesity-related traits have been noted as well in several studies [9]. In addition, linkage analysis in both rodent models and humans have found evidence of sex-specific loci affecting obesity-related traits $[10,11]$. Framingham Heart Study investigators found widespread evidence for sexspecific effects of genetic loci on body mass index, identifying several chromosomal regions with suggestive linkage to BMI in one sex, but not the other [11]. Indeed some effects were only seen in sex-stratified analyses and were not at all evident in the combined cohort of men and women. More recently, two genome-wide association study meta-analyses of WHR examined their top loci for sex differences and identified sexspecific effects for several loci $[8,12]$.

We sought evidence for significant differences in SNP effects on adiposity traits in men and women across the 
genome by carrying out a genome-wide association study modeling gene by sex interaction for WHR, WC, and BMI in the population-based Framingham Heart Study. Genomewide association analysis of SNPs having main effects (as opposed to gene by sex interaction) on obesity were reported earlier in the Framingham Heart Study using $100 \mathrm{~K}$ SNPs, but gene by sex interactions were not considered at that time [13]. Subsequently, the full genotype data $(>500 \mathrm{~K}$ SNPs) have been pooled with other studies and reported in large meta-analyses, which found evidence of gene by sex interaction for WHR but not BMI among the SNPs with main effects $[5,8]$.

\section{Materials and Methods}

2.1. Study Population. We conducted this research using data from the Framingham Heart Study, a population-based, longitudinal study of families living in the town of Framingham, Massachusetts collected over three-generations beginning in 1948. An overview of the study is provided at the dbGap website (http://www.ncbi.nlm.nih.gov/sites/entrez?db=gap) and detailed descriptions are available elsewhere $[14,15]$. Briefly, the original study (Generation 1) enrolled 5209 individuals, primarily Caucasian, and it later added the offspring of the original cohort (Generation 2), and the grandchildren (Generation 3 ) of the original cohort. Primary analyses were carried out using data from the five first exams of subjects in Generation 2, collected between 1971 and 1994. Obesity-related traits evaluated in this study included BMI, measured at exams 1, 2, 3, 4, and 5, WHR, measured at exams 4 and 5, and WC measured at exams 4 and 5 . We limited our analyses to these exams due to a drop in sample size at subsequent exams. Replication of genome wide association study (GWAS) results was sought in subjects from Generation 3 (data collected in 2002-2005).

Individuals with diabetes $(n=92,94,59,27,116$, and 136 for generation 2 exams 1, 2, 3, 4, 5 and generation 3 exam 1, resp.) or thyroid disorder ( $n=117,94,9,36,265$, and 72 for generation 2 exams 1, 2, 3, 4, 5 and generation 3 exam 1 , resp.) were removed because these diseases have an effect on both BMI and fat distribution. The data were further trimmed, excluding individuals with outlier trait values determined by taking the mean of the phenotype (independently for each exam and each sex) and adding/subtracting three standard deviations. Removal of outlier values in the BMI GWAS data was performed with weight, height, and BMI. WC and hip circumference (HC) outliers were also eliminated in the waist phenotype GWAS. Finally, we restricted our analysis to premenopausal women and individuals under the age of 50 to enhance differences related to estrogen-mediated gene by sex interaction and to reduce as much as possible the age-related differences in association that may occur across exams. The total sample sizes for the BMI GWAS after genotype quality control and trait outlier removal were $3150,1991,1630,1330,990$, and 2872 for generation 2 exams $1,2,3,4,5$, and generation 3 exam 1 , respectively. The sample sizes for the waist phenotype GWAS were 1330, 984, and 2872 for generation 2 exams 4, 5 and generation 3 exam 1, respectively.
2.2. Genotype Data and Quality Control. Genome-wide genotypes and detailed clinical data have been made accessible to the research community through the SHARe project (SNP-Health Association Resource). The study protocol was approved by Duke University's Institutional Review Board and the Framingham SHARe Data Access Committee. The unfiltered genotype data contained 9215 individuals (all generations) genotyped for 549782 SNPs. This included 500568 SNPs from the Affymetrix $500 \mathrm{~K}$ mapping array and 49214 SNPs from the Affymetrix $50 \mathrm{~K}$ supplemental array (Affymetrix, Santa Clara, CA, USA). We used the toolset PLINK [16] to perform quality control. Individuals were excluded if genotyping rates were less than $97 \%$. Markers were excluded if genotyping rates were less than 97\%, minor allele frequencies were less than 0.05 , or if HardyWeinberg $P$-values were less than .001. All SNP exclusions were made sequentially in the preceding order. Using this filtered data, we checked for Mendel errors using a 5\% cutoff per family, and a 10\% cutoff per SNP (as defined in PLINK), but none were detected. Individuals were also excluded if the predicted sex based on X-chromosome genotypes did not match the recorded sex. Pairwise identity-by-descent measures were calculated to detect replicated samples and unknown interfamilial relationships. We detected 4 identical twins and randomly selected one member of each pair for the analytic sample. After quality controls, the remaining sample consisted of genotype data on 360811 SNPs, attaining a genotyping rate of $99.5 \%$.

2.3. Statistical Analyses. Analysis of WHR and WC were based on data obtained at exam $4(n=1330)$ and exam $5(n=984)$ of subjects from Generation 2 . The gene by sex GWAS was run on data from each exam separately. We ran the full model for both WHR and WC regressed on BMI, age, age-squared, genotype, sex, and the genotypeby-sex cross product. BMI was available at all exams, with adequate sample sizes on the first five exams. Five separate GWAS were run using the full model of BMI regressed on age, age-squared, genotype, sex, and the genotype-bysex cross product-one each for exams 1, 2, 3, 4, and 5 of Generation 2. SNPs were evaluated for associations in an additive genetic model. A main effect GWAS was also run for BMI across the five exams, using the model specifications above without the cross product term. Sexspecific associations were tested using the full model of BMI regressed on age, age-squared, and genotype on each sex. To account for relatedness, we used generalized estimating equations while accounting for sibling correlation in the Yags package [17] of the $R$ statistical language. The $P$ values of the covariates were obtained via the Wald test using robust standard errors. The Framingham population has been studied extensively, and evidence for considerable population stratification has not been detected. To test this assumption, we estimated the inflation factor by dividing the median of the observed $\chi^{2}$ statistics for each GWAS, by the expected median in the absence of stratification (0.456) $[18,19]$. Also, adjusted for population stratification with the scores of the first 10 principal components, computed with Eigenstrat [20]. We defined genome-wide significance using 
a Bonferroni cutoff of $1.4 \times 10^{-7}$, which corrects for 360811 tests.

Following genome-wide analysis, we annotated results using the WGAViewer package [21], Ensembl [22], and the UCSC genome browser. We generated plots using the Gap package [23] of the $R$ statistical language and Haploview software [24].

To enrich for true positive associations, we took a strategy whereby associations that appeared in all exams were considered to have a higher likelihood of being true associations. We expected earlier exams to have greater power due to larger sample sizes, but other factors, including decreased heritability with age [11] may affect the results as well. This strategy required us to make some decisions about what cutoff to use when comparing results across exams. We took the consensus across exams of the top most significant $10,100,1000$, and 10000 hits and found $0,0,4$, and 105 SNPs, respectively, and focused on the four SNPs from the top 1000 consensus further.

\section{Results}

Characteristics of the subjects from Generations 2 and 3 of the Framingham Study used in the current analyses are presented in Table 1, broken down by exam. For each exam, we restricted our analyses to men and women $<50$ years of age, resulting in a decrease in sample size over time, above and beyond the loss due to death or nonparticipation.

3.1. Genome-Wide Association Analysis of Gene by Sex Interaction for WHR and WC and BMI. None of the gene-bysex interaction GWAS revealed genome-wide significant loci. For BMI we noted marked heterogeneity in quantile-quantile (QQ) plots between exams (Figure 1), which does not appear to be a function of sample size (which decreases with exam). There is also some evidence of inflation in the QQ plots, which was not alleviated after controlling for population stratification. In sex-stratified analysis, the inflation appeared to be restricted to men. The top 1000 hits from each exam for each trait (ordered by the $P$-value of the gene by sex interaction term) were extracted (Supplementary Tables S1, S2, and S3 available online on doi:10.1155/2011/329038), and the intersection of those datasets was sought for each trait.

For WHR, we identified 43 SNPs (28 unique loci) and for WC, we identified 43 SNPs (27 unique loci) appearing among the top 1000 in both exams 4 and 5 (Tables S2 and S3). When examining loci across these two traits, SNPs near SPOCK3, OSTF1, RAB31, and RPF1 appear in the top 1000 consensus for WC and WHR. SPOCK3 stands out as appearing among the top 100 hits across both exams 4 and 5 for WC $(P=$ $5.33 \times 10^{-7}$ and $\left.P=2.45 \times 10^{-5}\right)$ and WHR $\left(P=1.85 \times 10^{-4}\right.$ and $P=7.95 \times 10^{-5}$ ).

For BMI, only four SNPs appeared among the top 1000 hits in all five exams. All four SNPs localized to the same linkage disequilibrium block on chromosome $1, \sim 100 \mathrm{~kb}$ downstream of LYPLAL1. Supplementary Table S3.6 shows the location, minor allele frequency, $P$ values, and rank of each SNP by sex interaction by exam. We were most intrigued

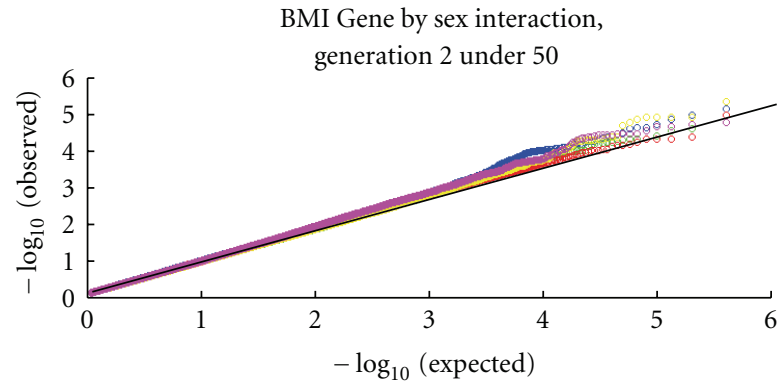

(a)

BMI main effect, generation 2 under 50

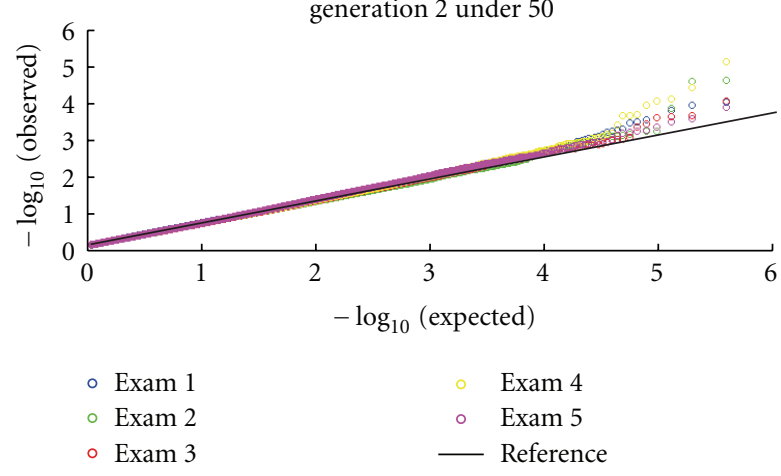

(b)

FIGURE 1: QQ plots for gene by sex interaction (a) and main effect (b) GWAS for body mass index (BMI) in Generation 2, exams 1, 2, 3,4 , and 5 .

by these findings as the LYPLAL1 locus has been reported as a sex-specific locus affecting central adiposity in two prior genome-wide association meta-analyses $[8,12]$. The extent of linkage disequilibrium (LD) surrounding the associated SNPs in the region of LYPLAL1 was determined in the Hap Map phase $3 \mathrm{CEU}$ population by identifying the farthest SNP away in each direction that had $r^{2}>0.5$ for each of the four SNPs. The LD block extends over $330 \mathrm{~kb}$ from position $217,321,833$ to $217,655,426$, and encompasses the LYPLAL1 gene (Figure 2). The block does not include the SNPs from Lindgren et al. [12] or Heid et al. [8], which are in moderate linkage disequilibrium with each other and located an additional $55 \mathrm{~kb}$ and $258 \mathrm{~kb}$ downstream of LYPLAL1, respectively.

3.2. Replication of LYPLAL1 SNP Association with BMI in Framingham Generation 3 Subjects. We next sought to replicate the observed association in subjects from Generation 3 of the Framingham Study. Again, we restricted our analyses to those less than 50 years of age. A comparison of results by sex in the five exams of Generation 2 and in Generation 3 are shown in Figure 3 for the top associated LYPLAL1 SNP. The SNP by sex interaction for LYPLAL1 was significant in all Generation 2 exams, but not significant in Generation 3 subjects. However, when stratified by sex, the minor allele showed a consistent increase in BMI in men across generations (Figure 3). In contrast, in women the minor 
TABLE 1: Mean \pm standard deviation for obesity-related traits in Framingham subjects $<50$ years old.

\begin{tabular}{|c|c|c|c|c|c|c|c|}
\hline & Population & $\begin{array}{c}\text { Generation } 2, \\
\text { exam } 1\end{array}$ & $\begin{array}{c}\text { Generation } 2, \\
\text { exam } 2\end{array}$ & $\begin{array}{c}\text { Generation 2, } \\
\text { exam } 3\end{array}$ & $\begin{array}{c}\text { Generation } 2, \\
\text { exam } 4\end{array}$ & $\begin{array}{c}\text { Generation 2, } \\
\text { exam } 5\end{array}$ & $\begin{array}{c}\text { Generation 3, } \\
\text { exam } 1\end{array}$ \\
\hline \multirow{3}{*}{$\begin{array}{l}\text { Sample } \\
\text { size }(N)\end{array}$} & All & 3150 & 1991 & 1630 & 1330 & 990 & 2872 \\
\hline & Men & 1478 & 958 & 776 & 640 & 463 & 1388 \\
\hline & Women & 1672 & 1033 & 854 & 690 & 527 & 1484 \\
\hline \multirow{3}{*}{$\begin{array}{l}\text { Age } \\
\text { (years) }\end{array}$} & All & $33.71 \pm 8.59$ & $38.39 \pm 6.70$ & $40.46 \pm 5.77$ & $42.27 \pm 5.11$ & $43.68 \pm 4.44$ & $37.63 \pm 7.27$ \\
\hline & Men & $33.81 \pm 8.63$ & $38.38 \pm 6.88$ & $40.47 \pm 5.92$ & $42.14 \pm 5.23$ & $43.56 \pm 4.65$ & $37.79 \pm 7.30$ \\
\hline & Women & $33.62 \pm 8.56$ & $38.39 \pm 6.54$ & $40.45 \pm 5.63$ & $42.40 \pm 5.00$ & $43.78 \pm 4.24$ & $37.47 \pm 7.24$ \\
\hline \multirow{3}{*}{$\begin{array}{l}\mathrm{BMI}^{a} \\
\left(\mathrm{~kg} / \mathrm{m}^{2}\right)\end{array}$} & All & $24.85 \pm 3.77^{* * *}$ & $25.10 \pm 3.79^{* * * *}$ & $25.40 \pm 4.08^{* * *}$ & $25.97 \pm 4.37^{*}$ & $26.42 \pm 4.48$ & $26.23 \pm 4.70$ \\
\hline & Men & $26.42 \pm 3.47^{*}$ & $26.57 \pm 3.44^{* *}$ & $26.72 \pm 3.49^{* *}$ & $27.23 \pm 3.62$ & $27.55 \pm 3.92$ & $27.42 \pm 4.03$ \\
\hline & Women & $23.48 \pm 3.47^{* * *}$ & $23.74 \pm 3.59^{* * *}$ & $24.20 \pm 4.20^{* *}$ & $24.80 \pm 4.66^{*}$ & $25.42 \pm 4.70$ & $25.11 \pm 4.99$ \\
\hline \multirow{3}{*}{$\begin{array}{l}\text { Height } \\
(\mathrm{cm})\end{array}$} & All & $167.62 \pm 9.36^{* * * *}$ & $168.59 \pm 9.57^{* * *}$ & $169.66 \pm 9.20^{*}$ & $169.67 \pm 9.12^{*}$ & $169.56 \pm 9.06$ & $170.91 \pm 9.18$ \\
\hline & Men & $174.95 \pm 6.79^{* * * *}$ & $175.99 \pm 6.77^{* * *}$ & $176.75 \pm 6.66^{*}$ & $176.65 \pm 6.52 *$ & $176.69 \pm 6.41$ & $177.88 \pm 6.41$ \\
\hline & Women & $161.15 \pm 5.90^{* * * * *}$ & $161.73 \pm 6.02^{* * * *}$ & $163.23 \pm 5.86^{*}$ & $163.20 \pm 5.81$ & $163.29 \pm 5.85$ & $164.39 \pm 6.06$ \\
\hline \multirow{3}{*}{$\begin{array}{l}\text { Weight } \\
(\mathrm{kg})\end{array}$} & All & $69.77 \pm 14.52^{* * * *}$ & $71.25 \pm 14.62^{* * * *}$ & $72.89 \pm 14.97^{* * *}$ & $74.50 \pm 15.55^{* *}$ & $75.72 \pm 15.79$ & $76.32 \pm 16.55$ \\
\hline & Men & $80.26 \pm 11.76^{* * * *}$ & $81.63 \pm 11.34^{* * *}$ & $82.78 \pm 11.82^{* * *}$ & $84.27 \pm 12.24^{* *}$ & $85.34 \pm 13.02$ & $86.06 \pm 13.76$ \\
\hline & Women & $60.50 \pm 9.58^{* * * *}$ & $61.63 \pm 10.01^{* * * *}$ & $63.90 \pm 11.45^{* * *}$ & $65.45 \pm 12.51^{*}$ & $67.27 \pm 12.91$ & $67.20 \pm 13.47$ \\
\hline \multirow{3}{*}{$\mathrm{WC}^{b}(\mathrm{~cm})$} & All & - & - & - & $86.70 \pm 13.92^{* * * *}$ & $88.95 \pm 13.30^{* * *}$ & $91.03 \pm 13.33$ \\
\hline & Men & - & - & - & $95.85 \pm 9.93^{* *}$ & $96.49 \pm 10.34$ & $96.50 \pm 11.15$ \\
\hline & Women & - & - & - & $78.21 \pm 11.50^{* * * * *}$ & $82.36 \pm 12.04^{* * *}$ & $85.90 \pm 13.18$ \\
\hline \multirow{3}{*}{$\mathrm{HC}^{c}(\mathrm{~cm})$} & All & - & - & - & $100.07 \pm 9.08$ & $101.37 \pm 8.63$ & - \\
\hline & Men & - & - & - & $101.31 \pm 7.18$ & $101.83 \pm 6.85$ & - \\
\hline & Women & 一 & - & 一 & $98.92 \pm 10.42$ & $100.98 \pm 9.93$ & - \\
\hline \multirow{3}{*}{$\mathrm{WHR}^{d}$} & All & - & - & - & $0.86 \pm 0.10$ & $0.88 \pm 0.09$ & - \\
\hline & Men & - & - & - & $0.95 \pm 0.06$ & $0.95 \pm 0.06$ & - \\
\hline & Women & - & - & - & $0.79 \pm 0.06$ & $0.81 \pm 0.07$ & - \\
\hline
\end{tabular}

${ }^{a}$ Body mass index,

${ }^{b}$ Waist circumference,

${ }^{c}$ Hip circumference,

${ }^{d}$ Waist to hip ratio,

* Significant difference between Generation 2 and Generation 3 after controlling for age and age-squared $(* P<.001, * * P<1 e-5, * * * P<1 e-10, * * * * P<$ $1 e-20, * * * * * P<1 e-50)$. Significance in age differences is not noted.

allele was associated with lower BMI in Generation 2 but not in Generation 3.

3.3. Association of LYPLAL1 SNPs with Obesity-Related Traits. To understand the relationship between LYPLAL1 SNPs and obesity in greater detail, we examined the top SNP from the present study (rs7552206) along with SNPs from the Lindgren et al. [12] and Heid et al. [8] studies for association with related phenotypes, including height, weight, WC, and WHR (Supplemental Table S4). The rs7552206 by sex interaction for BMI tracked with weight in all five exams, and with WC and HC in the two exams that had these data available. However, the waist and hip associations were completely or nearly completely attenuated when controlling for BMI. For rs2605100 (Lindgren et al. [12]), no compelling evidence of gene by sex interaction in central adiposity was found. Heid et al. [8] independently found a female-biased WHR association with LYPLAL1 (rs4846567), an SNP in moderate linkage disequilibrium with the Lindgren et al. SNP. We analyzed an available proxy for this SNP (rs2820446, Hap
Map CEU $r^{2}=1$ ) and found a borderline significant geneby-sex interaction with WHR $(P=.09$; Supplement S4).

3.4. Genome-Wide Association Analysis of Gene Main Effects for BMI. We also explored our cross-exam consensus approach for detecting significant main effects for BMI, using the same age-restricted datasets as the gene by sex interaction analyses. As with our gene by sex interaction analyses, the QQ plots show marked heterogeneity between exams (Figure 1) and modest inflation, which was not accounted for by population stratification. Only one SNP, located $\sim 26 \mathrm{~kb}$ upstream of DUSP10 on chromosome 1, appeared among the top 1000 hits (Supplement S5) in all five exams of Generation 2 and was borderline significant in Generation 3 subjects (Figure 4). Interestingly, this locus is approximately $2.4 \mathrm{Mb}$ away from the gene by sex interaction LYPLAL1 SNPs. No SNPs from prior genome-wide association studies of BMI showed up among our top 1000 consensus, including SNPs in the genes INSIG2, FTO [13, 25], and MC4R [26] (Figure 4). Surprisingly, the SNPs identified with the 


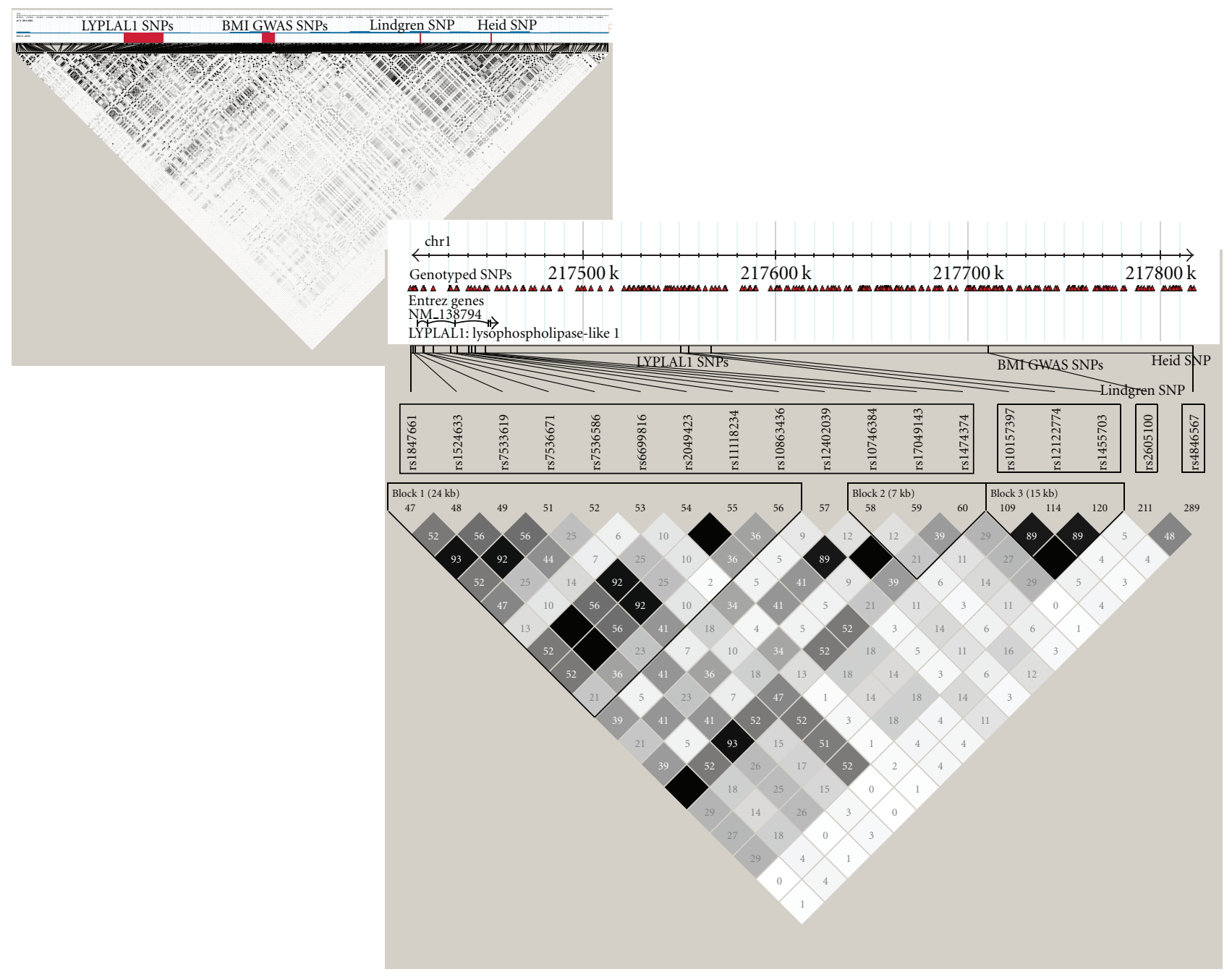

FIGURE 2: Linkage disequilibrium (shown as $r^{2}$ ) in the region encompassing LYPLAL1, the consensus SNPs associated with body mass index (BMI) in our gene by sex interaction GWAS, and the sex-specific SNPs associated with waist to hip ratio (WHR) in recent GWAS metaanalyses.

consensus approach yielded more significant $P$ values than other loci.

\section{Discussion}

We carried out a genome-wide assessment of gene by sex interaction for standard measures of obesity in men and women less than 50 years of age in the Framingham Heart Study. We took advantage of longitudinal data from multiple exams to identify loci showing consistent evidence of SNP by sex interaction across exams. Among the most prominent was a region $\sim 100 \mathrm{~kb}$ downstream of LYPLAL1, encoding the lysophospholipase-like 1 protein. We found evidence across five exams, spanning a 20-year time frame, of opposite effects of genetic variants in this region on BMI in men and women. An attempt to replicate this finding in a later generation of Framingham Heart Study subjects found a consistent, significant association in men, but not in women, possibly indicating a male-specific association.

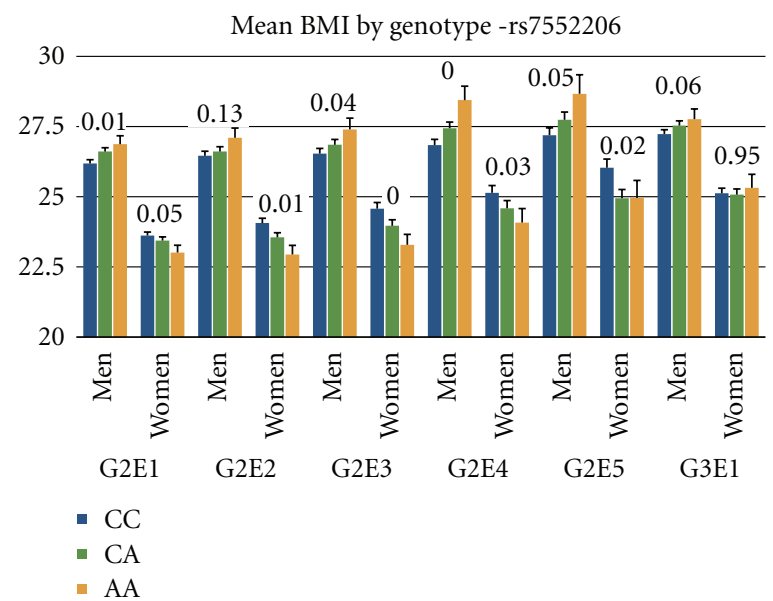

Figure 3: Mean body mass index (BMI) by genotype and sex across exams for the top associated SNP in LYPLAL1 (rs7552206) with Standard Error Bars and SNP P values. 
Ours is not the first study to link LYPLAL1 to obesity: two other genome-wide association meta-analyses identified this locus as having a sex-specific effect on WHR $[8,12]$. While neither SNP is in linkage disequilibrium with the region identified in our study, the coincidental discovery of two distinct regions near the LYPLAL1 locus associated with obesity-related traits in a sex-specific fashion warrants further attention. Moreover, a prior linkage analysis of BMI in Generation 2 of the Framingham Heart Study identified a male-biased linkage for BMI in the vicinity of LYPLAL1 on chromosome 1q41 [11]. None of the other sex-specific obsesity loci from Heid et al. [8] were found in our study.

LYPLAL1 is a member of the lysophospholipase gene family (EC number 3.1.1.5). It was initially identified as a gene on chromosome 1 found incidentally during investigation of a familial chromosomal translocation [27]. It was named on the basis of $\sim 30 \%$ predicted amino acid sequence homology with lysophospholipases I and II [28]. The sequence suggests an $\alpha / \beta$ hydrolase fold typically found in many lipases and esterases. LYPLAL1 was subsequently identified as one of 23 esterolytic/lipolytic proteins extracted from mouse adipose tissue. The presence of an active site serine was determined by activity tagging with a fluorescent probe of broad specificity, resembling a single-chain carboxylic acid ester. Similar probes modeling triglyceride and cholesteryl ester did not tag LYPLAL1 [29]. LYPLAL1 protein has not yet been isolated, however, and its substrate specificity is unknown. Along with the gene for adipocyte triglyceride lipase and several others related to lipolysis, LYPLAL1 mRNA was expressed more abundantly in abdominal subcutaneous adipose tissue from obese versus lean humans [30].

Given the minimal characterization of LYPLAL1, we can only speculate about its sex-specific role in adiposity. It might be involved in triglyceride synthesis or lipolysis, similar to some of the proteins with which it is coexpressed [31]. If indeed it is a lysophospholipase, it might play a role along with autotaxin, a secreted phospholipase $D$, in regulating extracellular levels of lysophosphatidic acid in adipose tissue. Via specific G protein-coupled receptors, lysophosphatidic acid has been shown to have varying effects on adipocyte differentiation and growth [32-34]. Another possibility relates to the endocannabinoid system, which has been a recent pharmacologic target for investigative obesity treatments. The monoglyceride, 2-arachidonoyl glycerol, as well as other esters or amides of long-chain polyunsaturated fatty acids belong to a family of compounds that are natural ligands for cannabinoid receptors. These endogenous signaling molecules affect physiologic and behavioral processes governing appetite and energy metabolism [31].

Interestingly lipolysis control has been shown to vary by sex in some studies [35] but not others [36]. The aforementioned study showing support for sex differences in lipolysis suggests that women show greater sensitivity to lipolysis in abdominal subcutaneous fat. The authors argue that the differences in lipolysis sensitivity are due to the presence of fewer inhibitory alpha-adrenergic receptors in the abdominal subcutaneous adipose tissue. This area

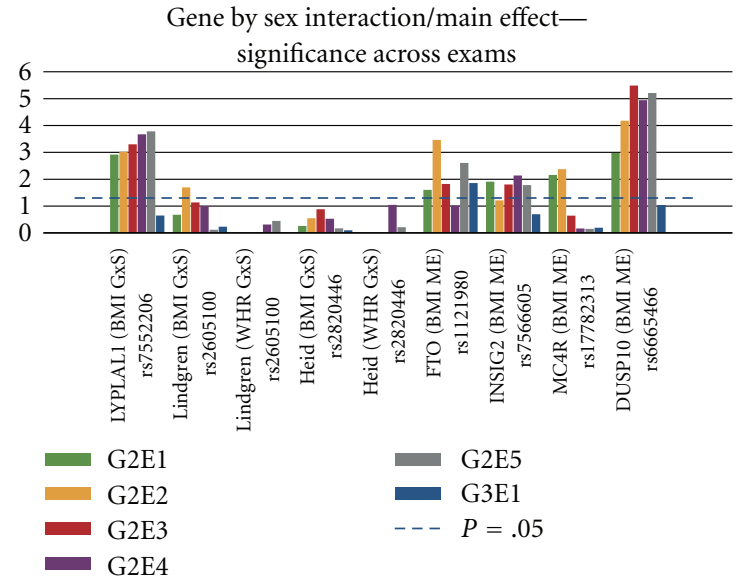

Figure 4: Significance level of main effect (ME) and/or gene by sex interaction $(\mathrm{GxS})$ associations with body mass index (BMI) and/or waist to hip ratio (WHR) for various loci of interest.

of lipid metabolism is not well understood, but recent discoveries and conflicting opinions warrant further studies on LYPLAL1 and its potential roles and sex-specific effects in lipid metabolism and obesity.

Our analysis revealed marked heterogeneity of effects across different exams of the study, both in gene by sex interaction and main effect analyses, even among established loci from other genome-wide association studies of BMI. The consensus approach appears to be robust, identifying a locus with strong prior evidence of gene by sex interaction for obesity-related traits. Using this approach, we also identified a possible novel candidate locus for BMI, located $\sim 26 \mathrm{~kb}$ upstream of DUSP10, encoding a dual specificity protein phosphatase. The DUSPs are a subclass of the protein tyrosine phosphatase gene superfamily that controls MAP kinase function [37].

Our study was carried out in the Framingham Heart Study Offspring cohort, a longitudinal, population-based study. Although no loci reached genome-wide significance in gene by sex interaction analyses, the longitudinal nature of the data allowed us to prioritize SNPs based on consistency of effect across exams. However, data on waist circumference were available only at two exams, limiting the effectiveness of our approach for these traits. Nonetheless, for BMI, this approach yielded a plausible candidate sex-specific locus and another sex-independent locus. Interestingly, in both of these cases, results from Generation 3 were not as significant as in Generation 2, possibly reflecting a cohort effect: Generation 2 subjects were enrolled nearly a decade or more prior to Generation 3 subjects. Generation 3 subjects were on average more overweight than Generation 2 subjects at comparable ages, consistent with temporal trends of increasing overweight/obesity observed in other populationbased studies. These differences, driven in large part by changes in diet and physical activity over time, may impact the heritability over time and thus, the ability to detect genetic effects. 


\section{Conclusions}

Few studies have systematically modeled gene by sex interaction for obesity-related traits on a genome-wide level. We confirm in our study that SNPs in the vicinity of LYPLAL1 may exhibit sex-specific effects on obesity-related traits. By utilizing a well-designed population-based study, and taking advantage of longitudinal data, we were able to demonstrate this effect using a much smaller sample size than the original meta-analysis that identified this locus. This has implications for the design of GWAS, where large samples sizes are often sought sometimes at the expense of population homogeneity. We suggest that smaller epidemiologically sound population-based studies may be more powerful than larger heterogeneous metacohorts. We also highlight the importance of considering longitudinal robustness of association within a cohort as another means of prioritizing loci and reducing false positive associations. Future studies of LYPLAL1 are needed to determine the basis of the apparent sex-specific effect on obesity.

\section{Acknowledgments}

The authors would like to thank the National Heart, Lung and Blood Institute (NHLBI) for creating the openaccess Framingham SHARe resource, and all of the study participants and Framingham Heart Study investigators who helped to create this tremendously valuable resource. The Framingham Heart Study is conducted and supported by the NHLBI in collaboration with Boston University. This manuscript was not prepared in collaboration with investigators of the Framingham Heart Study and does not necessarily reflect the opinions or views of the Framingham Heart Study, Boston University, or the NHLBI. This work was supported in part by National Institutes of Health Grants: RO1 HL085191 to J. McCarthy, PI. Jennifer Rowell was supported by NIH training Grant T32-DK007012-31 (Feinglos, PI), and Ashlee Benjamin by training Grant T32 GM071340 (Harer, PI).

\section{References}

[1] Y. Wang and M. A. Beydoun, "The obesity epidemic in the United States-gender, age, socioeconomic, racial/ethnic, and geographic characteristics: a systematic review and metaregression analysis," Epidemiologic Reviews, vol. 29, no. 1, pp. 6-28, 2007.

[2] QI. Zhang and Y. Wang, "Trends in the association between obesity and socioeconomic status in U.S. adults: 1971 to 2000," Obesity Research, vol. 12, no. 10, pp. 1622-1632, 2004.

[3] I. S. Okosun, S. T. Choi, J. M. Boltri et al., "Trends of abdominal adiposity in white, black, and Mexican-American adults, 1988 to 2000," Obesity Research, vol. 11, no. 8, pp. 1010-1017, 2003.

[4] I. S. Okosun, K. M.D. Chandra, A. Boev et al., "Abdominal adiposity in U.S. adults: prevalence and trends, 1960-2000," Preventive Medicine, vol. 39, no. 1, pp. 197-206, 2004.

[5] E. K. Speliotes, C. J. Willer, S. I. Berndt et al., "Association analyses of 249,796 individuals reveal 18 new loci associated with body mass index," Nature Genetics, vol. 42, no. 11, pp. 937-948, 2010.
[6] C. J. Willer, E. K. Speliotes, R. J. F. Loos et al., "Six new loci associated with body mass index highlight a neuronal influence on body weight regulation," Nature Genetics, vol. 41, no. 1, pp. 25-34, 2009.

[7] G. Thorleifsson, G. B. Walters, D. F. Gudbjartsson et al., "Genome-wide association yields new sequence variants at seven loci that associate with measures of obesity," Nature Genetics, vol. 41, no. 1, pp. 18-24, 2009.

[8] I. M. Heid, A. U. Jackson, J. C. Randall et al., "Metaanalysis identifies 13 new loci associated with waist-hip ratio and reveals sexual dimorphism in the genetic basis of fat distribution," Nature Genetics, vol. 42, no. 11, pp. 949-960, 2010.

[9] M. C. Zillikens, M. Yazdanpanah, L. M. Pardo et al., "Sexspecific genetic effects influence variation in body composition," Diabetologia, vol. 51, no. 12, pp. 2233-2241, 2008.

[10] S. Sammalisto, T. Hiekkalinna, K. Schwander et al., "Genomewide linkage screen for stature and body mass index in 3.032 families: evidence for sex- and population-specific genetic effects," European Journal of Human Genetics, vol. 17, no. 2, pp. 258-266, 2009.

[11] L. D. Atwood, N. L. Heard-Costa, C. S. Fox, C. E. Jaquish, and L. A. Cupples, "Sex and age specific effects of chromosomal regions linked to body mass index in the Framingham Study," BMC Genetics, vol. 7, article 7, 2006.

[12] C. M. Lindgren, I. M. Heid, J. C. Randall et al., "Genome-wide association scan meta-analysis identifies three loci influencing adiposity and fat distribution," PLoS Genetics, vol. 5, no. 6, Article ID e1000508, 2009.

[13] C. S. Fox, N. Heard-Costa, L. A. Cupples, J. Dupuis, R. S. Vasan, and L. D. Atwood, "Genome-wide association to body mass index and waist circumference: the Framingham Heart Study 100K project," BMC Medical Genetics, vol. 8, no. 1, article no. S18, 2007.

[14] D. R. Govindaraju, L. Adrienne Cupples, W. B. Kannel et al., "Chapter 2 genetics of the Framingham Heart Study population," Advances in Genetics, vol. 62, pp. 33-65, 2008.

[15] L. A. Cupples, H. T. Arruda, E. J. Benjamin et al., "The Framingham Heart Study 100K SNP genome-wide association study resource: overview of 17 phenotype working group reports," BMC Medical Genetics, vol. 8, no. 1, article no. S1, 2007.

[16] S. Purcell, B. Neale, K. Todd-Brown et al., "PLINK: a tool set for whole-genome association and population-based linkage analyses," American Journal of Human Genetics, vol. 81, no. 3, pp. 559-575, 2007.

[17] C. Vince, "yags: Yet Another GEE Solver. R package version 4.0-2," 2004.

[18] B. Devlin and K. Roeder, "Genomic control for association studies," Biometrics, vol. 55, no. 4, pp. 997-1004, 1999.

[19] S. A. Bacanu, B. Devlin, and K. Roeder, "Association studies for quantitative traits in structured populations," Genetic Epidemiology, vol. 22, no. 1, pp. 78-93, 2002.

[20] A. L. Price, N. J. Patterson, R. M. Plenge, M. E. Weinblatt, N. A. Shadick, and D. Reich, "Principal components analysis corrects for stratification in genome-wide association studies," Nature Genetics, vol. 38, no. 8, pp. 904-909, 2006.

[21] D. Ge, K. Zhang, A. C. Need et al., "WGA Viewer: software for genomic annotation of whole genome association studies," Genome Research, vol. 18, no. 4, pp. 640-643, 2008.

[22] T. J. P. Hubbard, B. L. Aken, S. Ayling et al., "Ensembl 2009," Nucleic Acids Research, vol. 37, no. 1, pp. D690-D697, 2009.

[23] J. H. Zhao, "Gap: genetic analysis package," Journal of Statistical Software, vol. 23, no. 8, pp. 1-18, 2007. 
[24] J. C. Barrett, B. Fry, J. Maller, and M. J. Daly, "Haploview: analysis and visualization of LD and haplotype maps," Bioinformatics, vol. 21, no. 2, pp. 263-265, 2005.

[25] A. Herbert, N. P. Gerry, M. B. McQueen et al., "A common genetic variant is associated with adult and childhood obesity," Science, vol. 312, no. 5771, pp. 279-283, 2006.

[26] F. Renström, F. Payne, A. Nordström et al., "Replication and extension of genome-wide association study results for obesity in 4923 adults from northern Sweden," Human Molecular Genetics, vol. 18, no. 8, pp. 1489-1496, 2009.

[27] D. David, J. Cardoso, B. Marques et al., "Molecular characterization of a familial translocation implicates disruption of HDAC9 and possible position effect on TGF $\beta 2$ in the pathogenesis of Peters' anomaly," Genomics, vol. 81, no. 5, pp. 489-503, 2003.

[28] A. Wang and E. A. Dennis, "Mammalian lysophospholipases," Biochimica et Biophysica Acta, vol. 1439, no. 1, pp. 1-16, 1999.

[29] R. Birner-Gruenberger, H. Susani-Etzerodt, M. Waldhubert et al., "The lipolytic proteome of mouse adipose tissue," Molecular and Cellular Proteomics, vol. 4, no. 11, pp. 1710 1717, 2005.

[30] G. R. Steinberg, B. E. Kemp, and M. J. Watt, "Adipocyte triglyceride lipase expression in human obesity," American Journal of Physiology, vol. 293, no. 4, pp. E958-E964, 2007.

[31] R. Zechner, P. C. Kienesberger, G. Haemmerle, R. Zimmermann, and A. Lass, "Adipose triglyceride lipase and the lipolytic catabolism of cellular fat stores," Journal of Lipid Research, vol. 50, no. 1, pp. 3-21, 2009.

[32] C. Pagès, A. Girard, O. Jeanneton et al., "LPA as a paracrine mediator of adipocyte growth and function," Annals of the New York Academy of Sciences, vol. 905, pp. 159-164, 2000.

[33] L. A. van Meeteren and W. H. Moolenaar, "Regulation and biological activities of the autotaxin-LPA axis," Progress in Lipid Research, vol. 46, no. 2, pp. 145-160, 2007.

[34] M. F. Simon, D. Daviaud, J. P. Pradère et al., "Lysophosphatidic acid inhibits adipocyte differentiation via lysophosphatidic acid 1 receptor-dependent down-regulation of peroxisome proliferator-activated receptor $\gamma 2$," Journal of Biological Chemistry, vol. 280, no. 15, pp. 14656-14662, 2005.

[35] C. M. Williams, "Lipid metabolism in women," Proceedings of the Nutrition Society, vol. 63, no. 1, pp. 153-160, 2004.

[36] J. Bülow, K. Gjeraa, L. H. Enevoldsen, and L. Simonsen, "Lipid mobilization from human abdominal, subcutaneous adipose tissue is independent of sex during steady-state exercise," Clinical Physiology and Functional Imaging, vol. 26, no. 4, pp. 205-211, 2006.

[37] M. Camps, A. Nichols, and S. Arkinstall, "Dual specificity phosphatases: a gene family for control of MAP kinase function,” FASEB Journal, vol. 14, no. 1, pp. 6-16, 2000. 


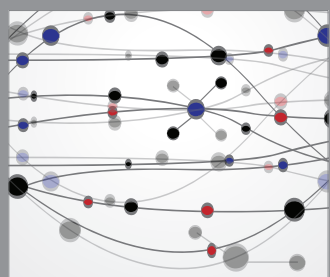

The Scientific World Journal
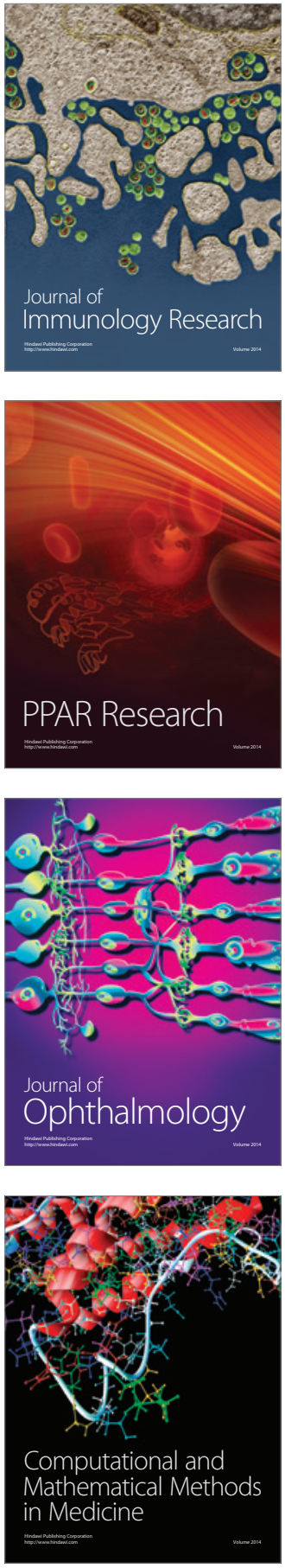

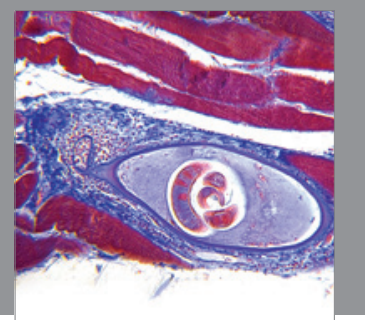

Gastroenterology

Research and Practice
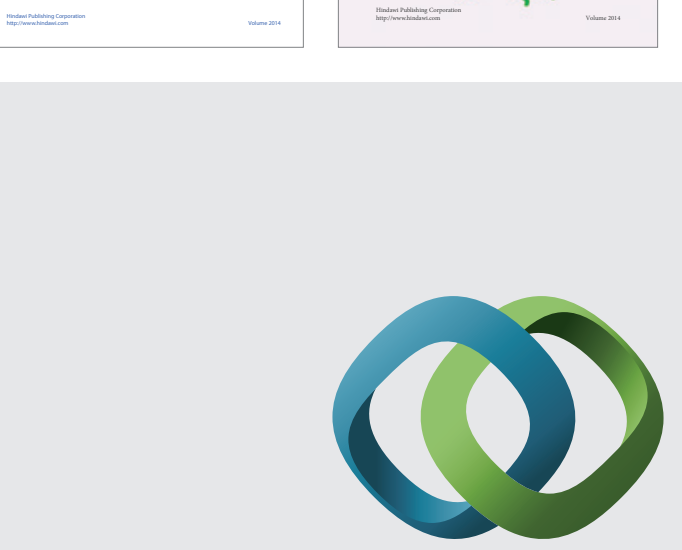

\section{Hindawi}

Submit your manuscripts at

http://www.hindawi.com
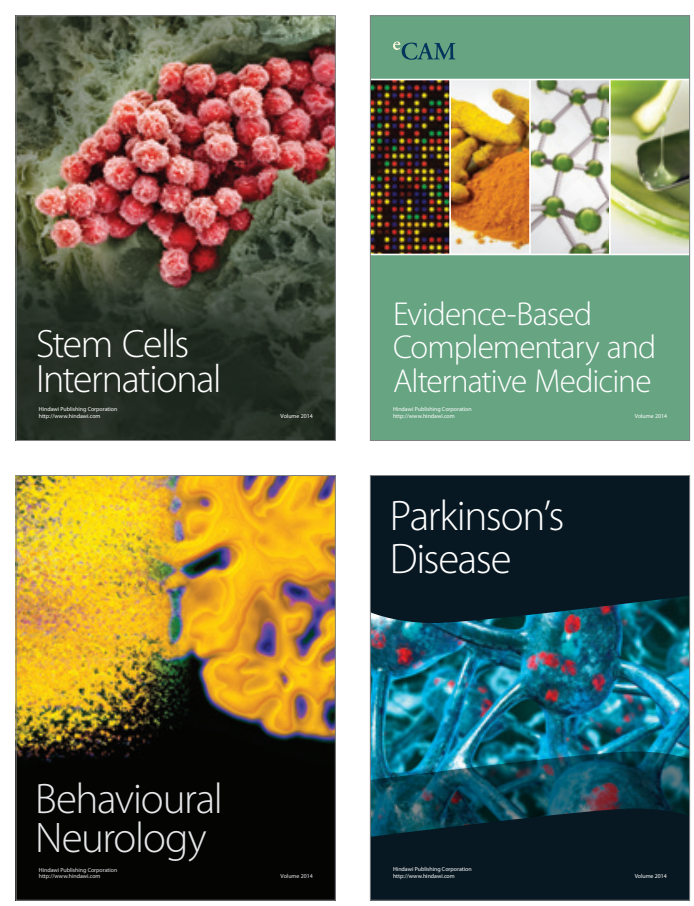

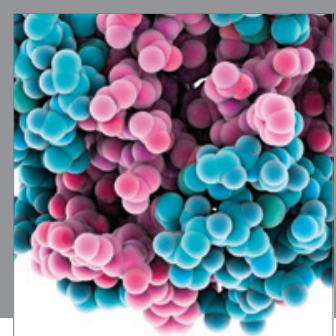

Journal of
Diabetes Research

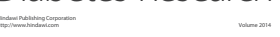

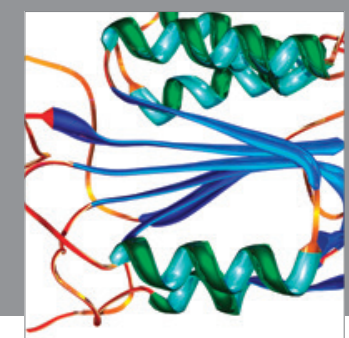

Disease Markers
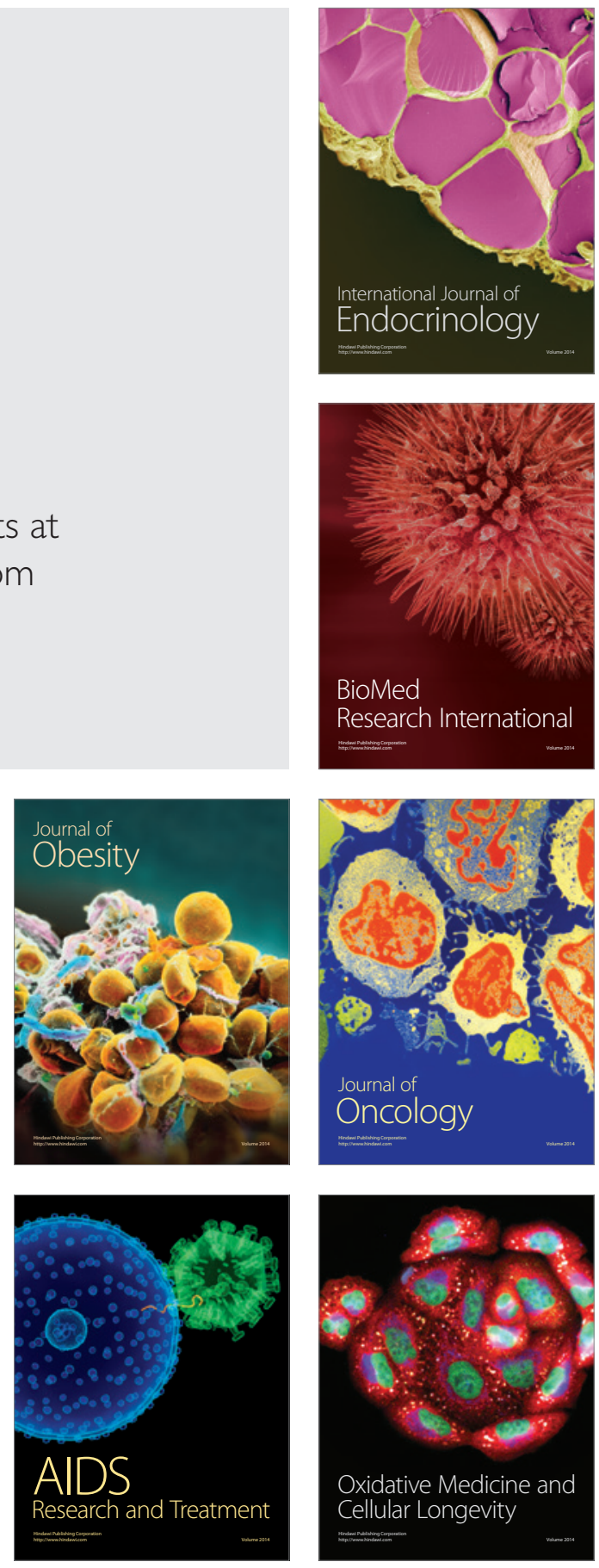